九州大学学術情報リポジトリ

Kyushu University Institutional Repository

\title{
DESIGN OF BIORTHOGONAL WAVELET FILTERS USING DYADIC LIFTING SCHEME
}

Abdukirim, Turghunjan

Department of Informatics, Kyushu University

Ni i jima, Koichi

Department of Informatics, Kyushu University

Takano, Shigeru

Department of Informatics, Kyushu University

https://doi.org/10.5109/12595

出版情報: Bulletin of informatics and cybernetics. 37, pp.123-136，2005-12. Research Association of Statistical Sciences

バージョン：

権利関係 : 


\title{
DESIGN OF BIORTHOGONAL WAVELET FILTERS USING DYADIC LIFTING SCHEME
}

\author{
by \\ Turghunjan Abdukinim, Koichi Niıjima and Shigeru TaKano
}

Reprinted from the Bulletin of Informatics and Cybernetics

Research Association of Statistical Sciences, Vol.37
FUKUOKA, JAPAN
2005




\title{
DESIGN OF BIORTHOGONAL WAVELET FILTERS USING DYADIC LIFTING SCHEME
}

\author{
By \\ Turghunjan Abdukirim, Koichi Niıjima $^{\dagger}$ and Shigeru TaKano ${ }^{\ddagger}$
}

\begin{abstract}
A method for designing biorthogonal wavelet filters is proposed. The method is used for constructing symmetric biorthogonal spline wavelet filters with finite length and vanishing moments. The constructed filters are applied together with a thresholding technique for removing Gaussian noise from noise corrupted images.
\end{abstract}

Key Words and Phrases: Biorthogonal wavelet filters, Dyadic lifting scheme, Finite length, Vanishing moment, Noise reduction.

\section{Introduction}

Orthogonal or biorthogonal wavelet transform has often been used in many image processing applications, because it makes possible multiresolution analysis and does not yield redundant information, see Mallat (1998). Daubechies (1992) has presented a constructive method of orthogonal wavelets with compact support. Unfortunately, such wavelets are not symmetric. The highly important linear phase constraint corresponding to symmetric wavelets may be maintained by relaxing the orthogonality constraint and using biorthogonal wavelets. Design of symmetric biorthogonal wavelet filters is an important issue in the area of image processing, because their associated wavelet transforms have good compression potential and low computational complexity. Unser and Aldroubi (1992) have presented polynomial spline wavelets which are biorthogonal wavelets and have been constructed using B-spline functions. Although these wavelets are smooth and symmetric, their filters do not have finite length.

In this paper, we describe how to design biorthogonal wavelet filters by using our lifting dyadic wavelet theory proposed in Abdukirim et al. (2003). Our method is used for constructing B-spline based biorthogonal wavelet filters with finite length and vanishing moments. The designed filters are different from those given in Unser and Aldroubi (1992). To confirm the efficiency of our filters, we apply them together with a softthresholding technique proposed by Donoho (1995) to remove Gaussian noise from noise corrupted images.

This paper is organized as follows. In Section 2, the definition of dyadic wavelet is given. We present one proposition concerning a dyadic lifting scheme in Section 3.

\footnotetext{
* Department of Informatics, Kyushu University 6-1 Kasuga-koen Kasuga Fukuoka 816-8580 Japan. tel +81-92-583-7637 t-abdu@i.kyushu-u.ac.jp

$\dagger$ Department of Informatics, Kyushu University 6-1 Kasuga-koen Kasuga Fukuoka 816-8580 Japan. tel +81-92-583-7631 niijima@i.kyushu-u.ac.jp

¥ Department of Informatics, Kyushu University 6-1 Kasuga-koen Kasuga Fukuoka 816-8580 Japan. tel +81-92-583-7641 takano@i.kyushu-u.ac.jp
} 
Section 4 describes a method for designing biorthogonal wavelet filters using the dyadic lifting scheme and a biorthogonal condition. We give a biorthogonal lifting scheme introduced by Sweldens (1996) in Section 5, which is needed for conducting filters with high vanishing moments. Section 6 applies our designing method for constructing Bspline based symmetric biorthogonal wavelet filters with finite length and vanishing moments. Section 7 concerns a noise reduction method by a combination of these filters with a thresholding technique. Simulation results are given in Section 8. Section 9 is a conclusion.

\section{Dyadic wavelet}

To design biorthogonal wavelet filters using a dyadic lifting scheme, we have to start from the description of dyadic wavelet.

Let $L^{2}(R)$ be the space of square integrable functions on real line $R$. We define the Fourier transform of the function $\psi \in L^{2}(R)$ by

$$
\hat{\psi}(\omega)=\int_{-\infty}^{+\infty} \psi(t) e^{-i \omega t} d t
$$

The definition of dyadic wavelet is as follows:

Definition. If there exist $A \geq 0$ and $B$ such that

$$
A \leq \sum_{j=-\infty}^{+\infty}\left|\hat{\psi}\left(2^{j} \omega\right)\right|^{2} \leq B
$$

then $\psi(t)$ is called a dyadic wavelet function.

From $(2)$, if $\psi(t)$ is dyadic, $\hat{\psi}(0)=0$ must be satisfied, i.e.

$$
\int_{-\infty}^{+\infty} \psi(t) d t=0
$$

To construct the dyadic wavelet function, we need a scaling function $\phi(t)$ satisfying a two-scale relation

$$
\phi(t)=\sum_{k} h[k] \sqrt{2} \phi(2 t-k) .
$$

The scaling function $\phi(t)$ is usually normalized as

$$
\int_{-\infty}^{+\infty} \phi(t) d t=1 .
$$

The Fourier transform of the scaling function (4) yields

$$
\hat{\phi}(\omega)=\frac{1}{\sqrt{2}} \hat{h}\left(\frac{\omega}{2}\right) \hat{\phi}\left(\frac{\omega}{2}\right),
$$

where $\hat{\phi}(\omega)$ is the same as given in $(1)$ and $\hat{h}(\omega)$ denotes a discrete Fourier transform

$$
\hat{h}(\omega)=\sum_{k} h[k] e^{-i \omega k}
$$


Since $\hat{\phi}(0)=1$, we can apply (5) and (6) to get $\hat{h}(0)=\sqrt{2}$ or $\sum_{k} h[k]=\sqrt{2}$. Using the scaling function $\phi(t)$ and the wavelet filter $g[k]$, we construct a dyadic wavelet function as

$$
\psi(t)=\sum_{k} g[k] \sqrt{2} \phi(2 t-k)
$$

Discrete dyadic wavelet transform of $f \in L^{2}(R)$ is defined using this function $\psi(t)$ by

$$
W f\left(n, 2^{j}\right)=\int_{-\infty}^{+\infty} f(t) \frac{1}{\sqrt{2^{j}}} \psi\left(\frac{t-n}{2^{j}}\right) d t,
$$

where $n$ and $j$ are integers.

Mallat (1998) presented a condition that enables us to expand $L^{2}(R)$-function by dyadic wavelet bases. To derive the reconstruction condition, we further need a dual scaling function and a dual wavelet function. The dual scaling function $\tilde{\phi}(t)$ is defined by using a two-scale relation

$$
\tilde{\phi}(t)=\sum_{k} \tilde{h}[k] \sqrt{2} \tilde{\phi}(2 t-k),
$$

and the dual wavelet function $\tilde{\psi}(t)$ is given by

$$
\tilde{\psi}(t)=\sum_{k} \tilde{g}[k] \sqrt{2} \tilde{\phi}(2 t-k) .
$$

Let us denote the discrete Fourier transforms of the filters $h[k], g[k], \tilde{h}[k]$, and $\tilde{g}[k]$ by $\hat{h}(\omega), \hat{g}(\omega), \hat{\tilde{h}}(\omega)$ and $\hat{\tilde{g}}(\omega)$, respectively. We suppose that these Fourier transforms satisfy the following condition

$$
\hat{\tilde{h}}(\omega) \hat{h}^{*}(\omega)+\hat{\tilde{g}}(\omega) \hat{g}^{*}(\omega)=2, \quad \omega \in[-\pi, \pi],
$$

where the symbol $*$ denotes complex conjugation. The condition (8) is called a reconstruction condition, and the filters $\hat{h}(\omega), \hat{g}(\omega), \hat{\tilde{h}}(\omega)$ and $\hat{\tilde{g}}(\omega)$ satisfying (8) dyadic wavelet filters.

\section{Dyadic dual lifting scheme}

As Sweldens (1996) introduced a lifting scheme for biorthogonal wavelet filters, we can construct a lifting scheme for the dyadic wavelet filters.

Proposition 1. Suppose that the discrete Fourier transforms $\hat{h}^{o}(\omega), \hat{g}^{o}(\omega), \hat{\tilde{h}}^{o}(\omega)$ and $\hat{\tilde{g}}^{o}(\omega)$ of the initial filters $h^{o}[k], g^{o}[k], \tilde{h}^{o}[k]$ and $\tilde{g}^{o}[k]$, respectively, satisfy the reconstruction condition (8). Then, the Fourier transforms $\hat{h}(\omega), \hat{g}(\omega), \hat{\tilde{h}}(\omega)$ and $\hat{\tilde{g}}(\omega)$ of dual lifting dyadic wavelet filters defined by

$$
\begin{aligned}
h[k] & =h^{o}[k]+\sum_{m} r[-m] g^{o}[k-m] \\
g[k] & =g^{o}[k], \\
\tilde{h}[k] & =\tilde{h}^{o}[k], \\
\tilde{g}[k] & =\tilde{g}^{o}[k]-\sum_{m} r[m] \tilde{h}^{o}[k-m]
\end{aligned}
$$


satisfy the reconstruction condition (8). Here $r[m]$ are lifting parameters. We call (9) a dyadic dual lifting scheme.

Proof. By the assumption, the Fourier transforms $\hat{h}^{o}(\omega), \hat{g}^{o}(\omega), \hat{\tilde{h}}^{o}(\omega)$ and $\hat{\tilde{g}}^{o}(\omega)$ satisfy (8), which can be written in the matrix form as

$$
\left(\begin{array}{ll}
\tilde{h}^{o}(\omega) & \hat{\tilde{g}}^{o}(\omega)
\end{array}\right)\left(\begin{array}{c}
\hat{h}^{o^{*}}(\omega) \\
\hat{g}^{o^{*}}(\omega)
\end{array}\right)=2 .
$$

The Fourier transforms of (9) are given by

$$
\begin{aligned}
& \hat{h}(\omega)=\hat{h}^{o}(\omega)+\hat{r}^{*}(\omega) \hat{g}^{o}(\omega), \\
& \hat{g}(\omega)=\hat{g}^{o}(\omega), \\
& \hat{\tilde{h}}(\omega)=\hat{\tilde{h}}^{o}(\omega), \\
& \hat{\tilde{g}}(\omega)=\hat{\tilde{g}}^{o}(\omega)-\hat{r}(\omega) \hat{\tilde{h}}^{o}(\omega) .
\end{aligned}
$$

We prove that (11) satisfies (8). Writing (11) in a matrix form and factorizing it, we obtain

$$
\begin{aligned}
& \left(\begin{array}{lll}
\hat{\tilde{h}}(\omega) & \hat{\tilde{g}}(\omega)
\end{array}\right)\left(\begin{array}{c}
\hat{h}^{*}(\omega) \\
\hat{g}^{*}(\omega)
\end{array}\right)
\end{aligned}
$$

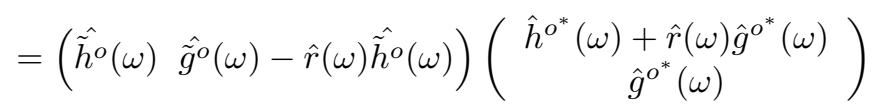

$$
\begin{aligned}
& =\left(\hat{\tilde{h}}^{o}(\omega) \hat{\tilde{g}}^{o}(\omega)\right)\left(\begin{array}{cc}
1 & -\hat{r}(\omega) \\
0 & 1
\end{array}\right)\left(\begin{array}{cc}
1 & \hat{r}(\omega) \\
0 & 1
\end{array}\right)\left(\begin{array}{c}
\hat{h}^{o^{*}}(\omega) \\
\hat{g}^{o^{*}}(\omega)
\end{array}\right)
\end{aligned}
$$

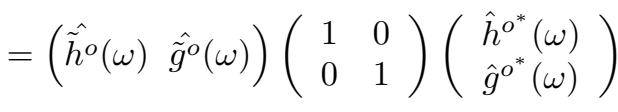

$$
\begin{aligned}
& =\left(\begin{array}{ll}
\tilde{h}^{o}(\omega) & \hat{\tilde{g}}^{o}(\omega)
\end{array}\right)\left(\begin{array}{c}
\hat{h}^{o^{*}}(\omega) \\
\hat{g}^{o^{*}}(\omega)
\end{array}\right) .
\end{aligned}
$$

A combination of this result with (10) proves that the Fourier transforms of (9) satisfy (8).

The dyadic dual lifting scheme is a systematic method for constructing a new analysis lowpass filter using known dyadic wavelet filters as building blocks.

\section{Biorthogonal condition in dyadic dual lifting scheme} condition

We assume that the filters $h[k], g[k], \tilde{h}[k]$ and $\tilde{g}[k]$ defined in Section 2 satisfy the

$$
\hat{\tilde{h}}(\omega) \hat{h}^{*}(\omega+\pi)+\hat{\tilde{g}}(\omega) \hat{g}^{*}(\omega+\pi)=0
$$


in addition to the reconstruction condition (8). Then, these filters are called biorthogonal wavelet filters. Using the wavelet function $\psi(t)$ with the biorthogonal wavelet filters, we define discrete biorthogonal wavelet transform

$$
d_{j}[n]=\int_{-\infty}^{+\infty} f(t) \frac{1}{\sqrt{2^{j}}} \psi\left(\frac{t}{2^{j}}-n\right) d t
$$

for an $L^{2}(R)$-function $f(t)$. Note that (13) is different from the discrete dyadic wavelet transform (7).

Mallat (1998) has derived a fast biorthogonal wavelet transform for computing $d_{j}[n]$ and its inverse, which are described in the following theorem.

Theorem 1. Under the conditions (8) and (12), we have

$$
\begin{array}{ll}
a_{j+1}[n]=\sum_{k} h[k-2 n] a_{j}[k], & j=0,1, \ldots, \\
d_{j+1}[n]=\sum_{k} g[k-2 n] a_{j}[k], & j=0,1, \ldots
\end{array}
$$

and

$$
a_{j}[k]=\sum_{n} \tilde{h}[k-2 n] a_{j+1}[n]+\sum_{n} \tilde{g}[k-2 n] d_{j+1}[n], \quad j=0,1, \ldots
$$

Here $a_{0}[n]$ is given by

$$
a_{0}[n]=\int_{-\infty}^{+\infty} f(t) \phi(t-n) d t
$$

and $a_{j+1}[n], j=0,1, \cdots$, represent a sequence of lowpass components.

In Theorem 1, the filters $h[k-2 n]$ and $g[k-2 n]$ are lowpass and highpass analysis filters, respectively, and the filters $\tilde{h}[k-2 n]$ and $g[k \sim 2 n]$ are lowpass and highpass synthesis filters, respectively. The algorithms (14) and (15) are decomposition formulae for one-dimensional signals. In case of image, first we apply them in the horizontal direction, and then in the vertical direction.

Now, we describe how to design the biorthogonal wavelet filters using the dyadic dual lifting scheme (9). By Proposition 1, (9) has already satisfied the reconstruction condition (8). We find a condition such that (9) fulfills the remaining biorthogonal condition (12).

Theorem 2. Suppose that the Fourier transforms $\hat{h}^{o}(\omega), \hat{g}^{o}(\omega), \hat{\tilde{h}}^{o}(\omega)$ and $\hat{\tilde{g}}^{o}(\omega)$ of the initial filters $h^{o}[k], g^{o}[k], \tilde{h}^{o}[k]$, and $\tilde{g}^{o}[k]$, respectively, satisfy the reconstruction condition (8). If the lifting parameters $r[m]$ satisfy the following condition

$$
\frac{\hat{\tilde{h}}^{o}(\omega) \hat{h}^{o^{*}}(\omega+\pi)+\hat{\tilde{g}}^{o}(\omega) \hat{g}^{o^{*}}(\omega+\pi)}{\tilde{h}^{o}(\omega) \hat{g}^{o^{*}}(\omega+\pi)}=2 \sum_{m} r[2 m+1] e^{-i(2 m+1) \omega},
$$

then the dyadic dual lifting scheme (9) becomes biorthogonal wavelet filters. Here the even lifting parameters $r[2 m]$ are free. 
Proof. Inserting (11), which is equivalent to (9), into (12), we obtain

$$
\begin{aligned}
\hat{\tilde{h}}^{o}(\omega) \hat{h}^{o^{*}}(\omega+\pi)+\hat{\tilde{g}}^{o}(\omega) \hat{g}^{o^{*}}(\omega+\pi) & \\
& +(\hat{r}(\omega+\pi)-\hat{r}(\omega)) \hat{\tilde{h}}^{o}(\omega) \hat{g}^{o^{*}}(\omega+\pi)=0 .
\end{aligned}
$$

On the other hand, we get

$$
\begin{aligned}
\hat{r}(\omega)-\hat{r}(\omega+\pi) & =\sum_{m}\left(1-(-1)^{m}\right) r[m] e^{-i m \omega} \\
& =2 \sum_{m} r[2 m+1] e^{-i(2 m+1) \omega} .
\end{aligned}
$$

Combining (18) with (19) yields the result of Theorem 2.

\section{Biorthogonal lifting scheme}

To design our biorthogonal wavelet filters, we need the lifting scheme for biorthogonal wavelet filters, which is described in the following proposition by Sweldens (1996).

Proposition 2. Suppose that the Fourier transforms $\hat{h}^{o}(\omega), \hat{g}^{o}(\omega), \hat{\tilde{h}}^{o}(\omega)$ and $\hat{\tilde{g}}^{o}(\omega)$ of the initial filters $h^{o}[k], g^{o}[k], \tilde{h}^{o}[k]$ and $\tilde{g}^{o}[k]$, respectively, are biorthogonal wavelet filters. Then, the Fourier transforms $\hat{h}(\omega), \hat{g}(\omega), \hat{\tilde{h}}(\omega)$ and $\hat{\tilde{g}}(\omega)$ of lifting biorthogonal wavelet filters defined by

$$
\begin{aligned}
h[k] & =h^{o}[k], \\
g[k] & =g^{o}[k]-\sum_{m} s[m] h^{o}[k-2 m], \\
\tilde{h}[k] & =\tilde{h}^{o}[k]+\sum_{m} s[-m] \tilde{g}^{o}[k-2 m], \\
\tilde{g}[k] & =\tilde{g}^{o}[k]
\end{aligned}
$$

become biorthogonal wavelet filters. Here $s[m]$ are lifting parameters. We call $(20)$ a biorthogonal lifting scheme.

The biorthogonal lifting scheme is a systematic method for constructing a new analysis highpass filter using known biorthogonal wavelet filters as building blocks.

\section{Design of B-spline based biorthogonal wavelet filters}

We apply Theorem 2 to design symmetric biorthogonal wavelet filters with finite length. We choose a B-spline function of first degree as a scaling function $\phi(t)$, because this function satisfies the two-scale relation (4). In Abdukirim et al. (2002) and Niijima et al. (2002), we constructed a B-spline based dyadic wavelet filter with 2 vanishing moments as follows. We choose lowpass analysis and synthesis filters $h[k]$ and $\tilde{h}[k]$ so that their Fourier transforms are given by

$$
\hat{h}(\omega)=\sqrt{2}\left(\cos \frac{\omega}{2}\right)^{2}
$$

and

$$
\hat{\tilde{h}}(\omega)=\hat{h}(\omega),
$$


respectively. The Fourier transform of highpass analysis filter $\hat{g}(\omega)$ is chosen as

$$
\hat{g}(\omega)=\sqrt{2} e^{-i \omega}\left(\sin \frac{\omega}{2}\right)^{2} .
$$

The Fourier transform of highpass synthesis filter $\tilde{g}[k]$ is restricted by the reconstruction condition (8) and has the form

$$
\hat{\tilde{g}}(\omega)=\sqrt{2} e^{-i \omega}\left(1+\left(\cos \frac{\omega}{2}\right)^{2}\right) .
$$

Table 1 lists the values of the filters in this case, where we put suffix ' $o$ ' on each filter, which stands for 'old'.

Table 1: Spline dyadic wavelet filters with 2 vanishing moments.

\begin{tabular}{|r|c|c|c|c|}
\hline$k$ & $\frac{h^{o}[k]}{\sqrt{2}}$ & $\frac{g^{o}[k]}{\sqrt{2}}$ & $\frac{\tilde{h}^{o}[k]}{\sqrt{2}}$ & $\frac{\tilde{g}^{o}[k]}{\sqrt{2}}$ \\
\hline-1 & 0.25 & & 0.25 & \\
0 & 0.50 & -0.25 & 0.50 & 0.25 \\
1 & 0.25 & 0.50 & 0.25 & 1.50 \\
2 & & -0.25 & & 0.25 \\
\hline
\end{tabular}

Since $\hat{h}^{o}(\omega), \hat{g}^{o}(\omega), \hat{\tilde{h}}^{o}(\omega)$ and $\hat{\tilde{g}}^{o}(\omega)$ satisfy

$$
\frac{\hat{\tilde{h}}^{o}(\omega) \hat{h}^{o *}(\omega+\pi)+\hat{\tilde{g}}^{o}(\omega) \hat{g}^{o *}(\omega+\pi)}{\hat{\tilde{h}}^{o}(\omega) \hat{g}^{o *}(\omega+\pi)}=2 e^{-i \omega},
$$

we can design biorthogonal wavelet filters using Theorem 2 . The condition that the lifting parameters $r[m]$ must satisfy is

$$
r[2 m+1]= \begin{cases}1, & m=0, \\ 0, & m \neq 0 .\end{cases}
$$

We determine the even lifting parameters $r[2 m]$ so that $r[m]$ are symmetric with respect to $m=1$ and satisfy vanishing moment conditions. The first vanishing moment condition of $r[2 m]$ is

$$
\sum_{m} r[2 m]=1
$$

The symmetry of $r[m]$ leads us to

$$
r[2 l]=r[-2 l+2]=a_{l}, \quad l=0, \pm 1, \ldots
$$

Example 1. We choose $a_{0}=a_{1}=1 / 2$ and $a_{l}=0, l \neq 0,1$. Since $\hat{g}^{o}(\omega)$ satisfies

$$
\begin{aligned}
& \left.\frac{d^{k} \hat{g}^{o}(\omega)}{d \omega^{k}}\right|_{\omega=0}=0, \quad 0 \leq k \leq 1, \\
& \left.\frac{d^{2} \hat{g}^{o}(\omega)}{d \omega^{2}}\right|_{\omega=0}=\frac{\sqrt{2}}{2},
\end{aligned}
$$


Table 2: Biorthogonal wavelet filters with 2 vanishing moments.

\begin{tabular}{|r|c|c|c|c|}
\hline$k$ & $\frac{h[k]}{\sqrt{2}}$ & $\frac{g^{o}[k]}{\sqrt{2}}$ & $\frac{\tilde{h}^{o}[k]}{\sqrt{2}}$ & $\frac{\tilde{g}[k]}{\sqrt{2}}$ \\
\hline-2 & -0.125 & & & \\
-1 & 0.250 & & 0.25 & -0.125 \\
0 & 0.750 & -0.25 & 0.50 & -0.250 \\
1 & 0.250 & 0.50 & 0.25 & 0.750 \\
2 & -0.125 & -0.25 & & -0.250 \\
3 & & & & -0.125 \\
\hline
\end{tabular}

we see from the result of Abdukirim et al. (2003) that the filter $g^{o}[k]$ has 2 vanishing moments. Thus, we obtain the biorthogonal wavelet filters with 2 vanishing moments as shown in Table 2 .

Example 2. We can design a highpass analysis filter with 3 or more vanishing moments using Proposition 2 and the result of Abdukirim et al. (2003). The condition that $\hat{s}(\omega)$ must satisfy is

$$
\begin{aligned}
& \left.\frac{d^{k} \hat{s}(2 \omega)}{d \omega^{k}}\right|_{\omega=0}=0, \quad 0 \leq k \leq 1, \\
& \left.\frac{d^{2} \hat{s}(2 \omega)}{d \omega^{2}}\right|_{\omega=0}=\frac{1}{8} .
\end{aligned}
$$

Considering this condition and symmetry of $\hat{s}(\omega)$, we choose

$$
\hat{s}(2 \omega)=\frac{1}{16} e^{-i \omega} \sin ^{2} \omega .
$$

Thus, we obtain a highpass analysis filter with 4 vanishing moments as shown in Table 3.

Table 3: Biorthogonal wavelet filters with 4 vanishing moments.

\begin{tabular}{|r|c|c|c|c|}
\hline$k$ & $\frac{h[k]}{\sqrt{2}}$ & $\frac{g[k]}{\sqrt{2}}$ & $\frac{h[k]}{\sqrt{2}}$ & $\frac{\tilde{g}[k]}{\sqrt{2}}$ \\
\hline-4 & & & -0.001953125 & \\
-3 & & -0.001953125 & -0.003906250 & \\
-2 & -0.125 & 0.003906250 & 0.015625000 & \\
-1 & 0.250 & 0.015625000 & 0.253906250 & -0.125 \\
0 & 0.750 & -0.253906250 & 0.472656250 & -0.250 \\
1 & 0.250 & 0.472656250 & 0.253906250 & 0.750 \\
2 & -0.125 & -0.253906250 & 0.015625000 & -0.250 \\
3 & & 0.015625000 & -0.003906250 & -0.125 \\
4 & & 0.003906250 & -0.001953125 & \\
5 & & -0.001953125 & & \\
\hline
\end{tabular}

Example 3. Let $a_{0}=a_{1}=5 / 8, a_{-} 1=a_{2}=-1 / 8$ and $a_{l}=0, l \neq 0, \pm 1,2$. Thus, 
we obtain biorthogonal wavelet filters as shown in Table 4 . Since $\hat{g}^{o}(\omega)$ satisfies

$$
\begin{aligned}
& \left.\frac{d^{k} \hat{g}^{o}(\omega)}{d \omega^{k}}\right|_{\omega=0}=0, \quad 0 \leq k \leq 1, \\
& \left.\frac{d^{2} \hat{g}^{o}(\omega)}{d \omega^{2}}\right|_{\omega=0}=\frac{\sqrt{2}}{2},
\end{aligned}
$$

the filter $g^{o}[k]$ shown in Table 4 has 2 vanishing moments.

Table 4: Biorthogonal wavelet filters with 2 vanishing moments.

\begin{tabular}{|r|c|c|c|c|}
\hline$k$ & $\frac{h[k]}{\sqrt{2}}$ & $\frac{g^{o}[k]}{\sqrt{2}}$ & $\frac{\tilde{h}^{o}[k]}{\sqrt{2}}$ & $\frac{\tilde{g}[k]}{\sqrt{2}}$ \\
\hline-4 & 0.03125 & & & \\
-3 & -0.06250 & & & 0.03125 \\
-2 & -0.12500 & & & 0.06250 \\
-1 & 0.31250 & & 0.25 & -0.12500 \\
0 & 0.68750 & -0.25 & 0.50 & -0.31250 \\
1 & 0.31250 & 0.50 & 0.25 & 0.68750 \\
2 & -0.12500 & -0.25 & & -0.31250 \\
3 & -0.06250 & & & -0.12500 \\
4 & 0.03125 & & & 0.06250 \\
5 & & & & 0.03125 \\
\hline
\end{tabular}

Example 4. Similarly in Example 2, we can design a highpass analysis filter with 3 or more vanishing moments using Proposition 2 and the result of Abdukirim et al. (2003). The condition that $\hat{s}(\omega)$ must satisfy is

$$
\begin{aligned}
& \left.\frac{d^{k} \hat{s}(2 \omega)}{d \omega^{k}}\right|_{\omega=0}=0, \quad 0 \leq k \leq 1, \\
& \left.\frac{d^{2} \hat{s}(2 \omega)}{d \omega^{2}}\right|_{\omega=0}=\frac{1}{8} .
\end{aligned}
$$

By this condition and the symmetry of $\hat{s}(\omega)$, we choose

$$
\hat{s}(2 \omega)=\frac{1}{16} e^{-i \omega} \sin ^{2} \omega
$$

Thus, highpass analysis filters with 4 vanishing moments are obtained, which are shown in Table 5 .

\section{Noise reduction}

We apply the filters designed in Section 6 to image noise reduction. We consider a discrete noise signal can be represented by the following formula

$$
y(n)=f(n)+\sigma e(n), \quad 0 \leq n \leq N-1 .
$$


Table 5: Biorthogonal wavelet filters with 4 vanishing moments.

\begin{tabular}{|c|c|c|c|c|}
\hline$k$ & $\frac{h[k]}{\sqrt{2}}$ & $\frac{g[k]}{\sqrt{2}}$ & $\frac{\tilde{h}[k]}{\sqrt{2}}$ & $\frac{\tilde{g}[k]}{\sqrt{2}}$ \\
\hline-6 & & 0.00048828125 & 0.00048828125 & \\
-5 & & -0.00097656250 & -0.00292968750 & \\
-4 & 0.03125 & -0.00292968750 & -0.00683593750 & 0.03125 \\
-3 & -0.06250 & -0.0156507575 & 0.06250 \\
-2 & -0.12500 & 0.00683593750 & 0.01513671875 & \\
-1 & 0.31250 & 0.01513671875 & 0.25585937500 & -0.12500 \\
0 & 0.68750 & -0.25585937500 & 0.47460937500 & -0.31250 \\
1 & 0.31250 & 0.47460937500 & 0.25585937500 & 0.68750 \\
2 & -0.12500 & -0.25585937500 & 0.01513671875 & -0.31250 \\
3 & -0.06250 & 0.01513671875 & -0.00683593750 & -0.12500 \\
4 & 0.03125 & 0.00683593750 & -0.00292968750 & 0.06250 \\
5 & & -0.00292968750 & 0.00097656250 & 0.03125 \\
6 & & -0.00097656250 & 0.00048828125 & \\
7 & & 0.00048828125 & & \\
\hline
\end{tabular}

Here $y(n)$ represents a noisy signal, $f(n)$ is an unknown deterministic signal, $e(n)$ is the standard Gaussian white noise, and $\sigma$ is a noise level. Noise reduction methods have been studied intensely in wavelet signal processing by Abdukirim et al. (2003) and Donoho (1995). In this paper, we employ a soft-thresholding method proposed in Donoho (1995) as a noise reduction technique.

Let $C_{0}[n, l]$ be an image corrupted by Gaussian white noise. Gaussian white noise $N(0,1)$ was generated by repeated sampling from $N(0,1)$. Applying the decomposition formulae (14) and (15) in the horizontal and the vertical directions to $C_{0}[n, l]$ successively, we compute the lowpass components $C_{j}[n, l]$ at $j$-th resolution level and three kinds of highpass components $D_{j}^{m}[n, l], 1 \leq m \leq 3$. Here the filters $h[k]$ and $g[k]$ in (14) and (15) mean the lifting biorthogonal filters constructed in Section 6. The highpass components $D_{j}^{m}[n, l], 1 \leq m \leq 3$ are changed in one-dimensional sequences $D_{j}^{m}[k]$, $1 \leq m \leq 3$, respectively, to apply the noise reduction method. The soft thresholding procedure for $D_{j}^{m}[k]$ is to compute

$$
D_{j}^{m, *}[k]=\operatorname{sgn}\left(D_{j}^{m}[k]\right) \max \left\{\left|D_{j}^{m}[k]\right|-\delta, 0\right\} .
$$

Here we choose the threshold $\delta$ as $\delta=\sigma \sqrt{2 \log _{e} N}$ following Donoho (1995), where the noise variance $\sigma^{2}$ is computed by

$$
\sigma^{2}=\operatorname{Var}\left\{D_{j}^{m}[k]\right\} / 0.6745, \quad 1 \leq m \leq 3,
$$

and $N$ is the number of pixels in the image.

The noise reduction is done as follows. First, we rearrange $D_{j}^{m, *}[k], 1 \leq m \leq 3$ obtained by the above procedure to get two-dimensional components $D_{j}^{m, *}[n, l], 1 \leq$ $m \leq 3$, respectively. Next, we reconstruct $C_{j-1}^{*}[n, l]$ by substituting these components and lowpass components $C_{j}[n, l]$ into a two-dimensional version of the inverse formula (16). Repeating this procedure, we can get an image $C_{0}^{*}[n, l]$ where Gaussian noise has been removed. 


\section{Simulation}

The simulations are conducted on two natural gray scale test images of size $512 \times$ 512. The biorthogonal wavelet transform employs biorthogonal wavelet filters designed in Section 6. Gaussian noise having the variances from 10 to 30 is added to Lena and Peppers images shown in Figures 1 (a) and 2 (a), respectively. The noise corrupted images are shown in Figures 1 (b) and 2 (b). The images were decomposed 3 times using the analysis filters listed in Tables 2 to 5 . Next, the soft thresholding technique presented in Section 7 was applied only to the highpass components. Finally, we reconstructed the images using (16) described in Section 4 and the synthesis filters listed in Tables 2 to 5. Simulation results are shown in Figures 1 (c,d,e,f) and 2 (c,d,e,f).

Image quality is characterized by a mean square error (MSE), which possesses the advantage of a simple mathematical structure. For a discrete signal $f(n)$ and its approximation $\tilde{f}(n), 0 \leq n \leq N-1$, the MSE is defined to be

$$
\mathrm{MSE}=\frac{1}{N} \sum_{n=0}^{N-1}[\tilde{f}(n)-f(n)]^{2} .
$$

To estimate the image quality, we used the MSE-based evaluation standard

\section{Conclusion}

$$
\mathrm{PSNR}=10 \log _{10}\left(\frac{255 \times 255}{\mathrm{MSE}}\right) .
$$

We proposed a design method of biorthogonal wavelet filters by using a lifting dyadic wavelet theory. The method was applied for constructing B-spline based symmetric biorthogonal wavelet filters with finite length and vanishing moments. These filters together with a soft-thresholding technique were applied to the reduction of Gaussian noise from noise corrupted images. Simulation results show good denoising effects of highpass analysis filters with vanishing moments. Applications of the filters to other kinds of image processing remain as a future work.

\section{References}

T. Abdukirim, S. Takano and K. Niijima. (2002). Construction of spline dyadic wavelet filters, Research Report on Information Science and Electrical Engineering of Kyushu University, 7, 1-6.

T. Abdukirim, K. Niijima and S. Takano. (2003). Lifting dyadic wavelets for Denoising, Proceedings of The 2003 International TICSP Workshop on Spectal Methods and Multirate Signal Processing, SMMSP2003, 147-154.

I. Daubechies. (1992). Ten Lectures on Wavelets, New York: SIAM Press.

D.L. Donoho. (1995). De-noising by soft- threshold, IEEE Trans. on Inform. Theory, 38, $613-627$.

S. Mallat. (1998). A Wavelet Tour of Signal Processing, Academic Press.

K. Niijima, T. Abdukirim and S. Takano. (2002). Enhancement and edge detection of images by spline dyadic wavelets, Proceedings of IWIE 2002: International Workshop on Information and Electrical Engineering, 33-38. 
W. Sweldens. (1996). The lifting scheme: A custom-design construction of bi-orthogonal wavelets, Appl. Comput. Harmon. Anal., 3, 186-200.

M. Unser and A. Aldroubi. (1992). Polynomial splines and wavelets-A signal processing perspective, Wavelets-A Tutorial in Theory and Applications, C.K.Chui(ed), Academic Press, New York, 91-122.

Received October 28, 2003

Revised July 23, 2004 


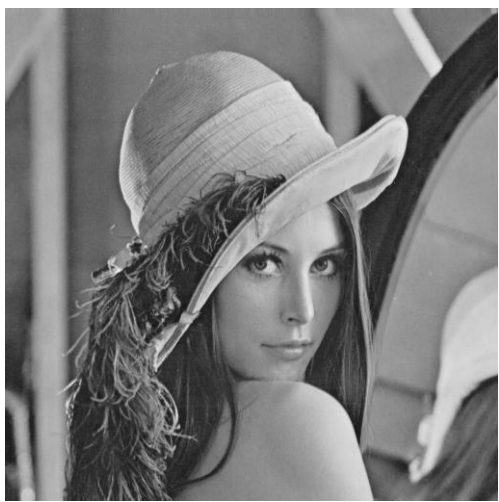

(a)
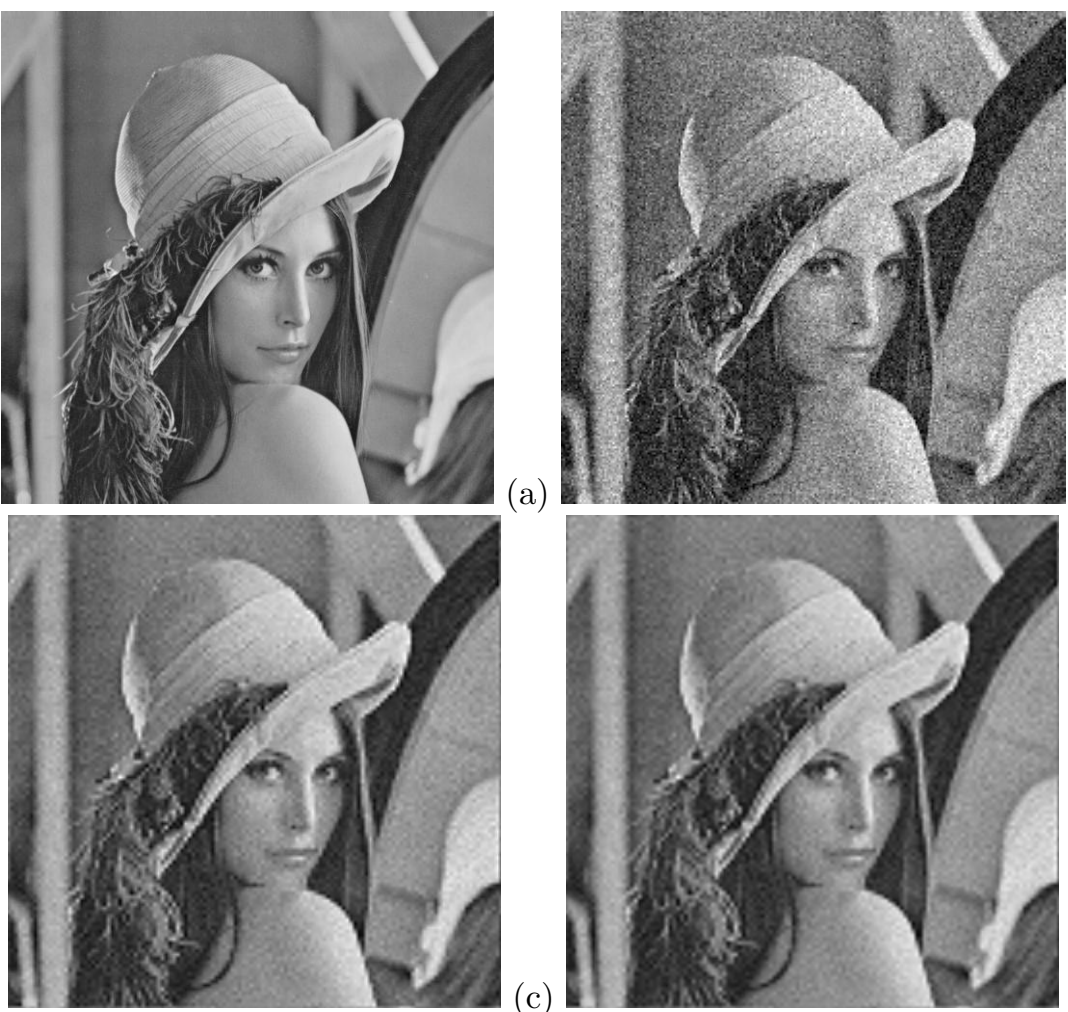

(b)
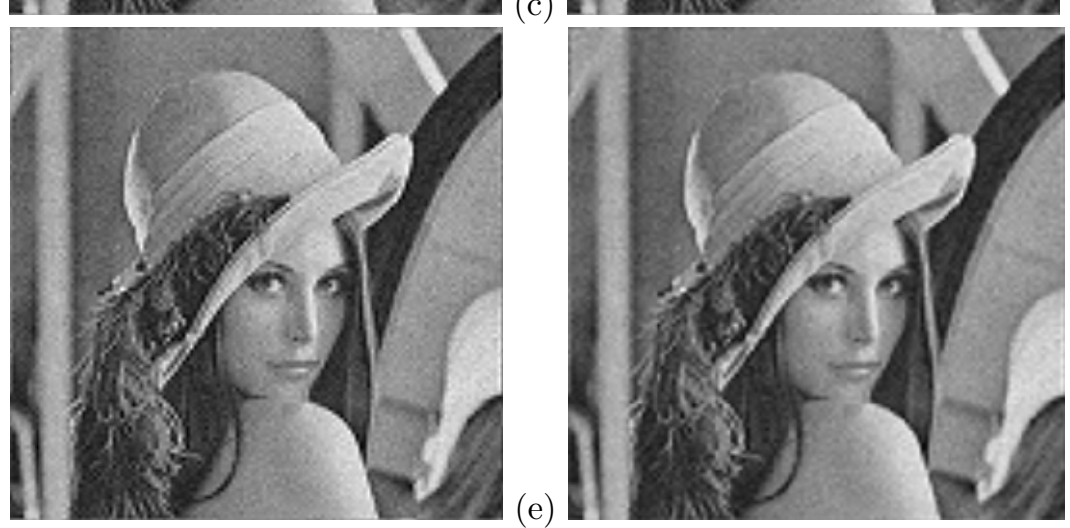

(d)

(e)

(f)

Figure 1: (a) Lena image, (b) noise corrupted image (PSNR=21.64dB), (c) an image restored by the filters listed in Table $2(\mathrm{PSNR}=27.52 \mathrm{~dB}),(\mathrm{d})$ an image restored by the filters listed in Table $3(\mathrm{PSNR}=27.74 \mathrm{~dB}),(\mathrm{e})$ an image restored by the filters listed in Table $4(\mathrm{PSNR}=27.74 \mathrm{~dB})$, (f) an image restored by the filters listed in Table 5 $(\mathrm{PSNR}=28.00 \mathrm{~dB})$. 

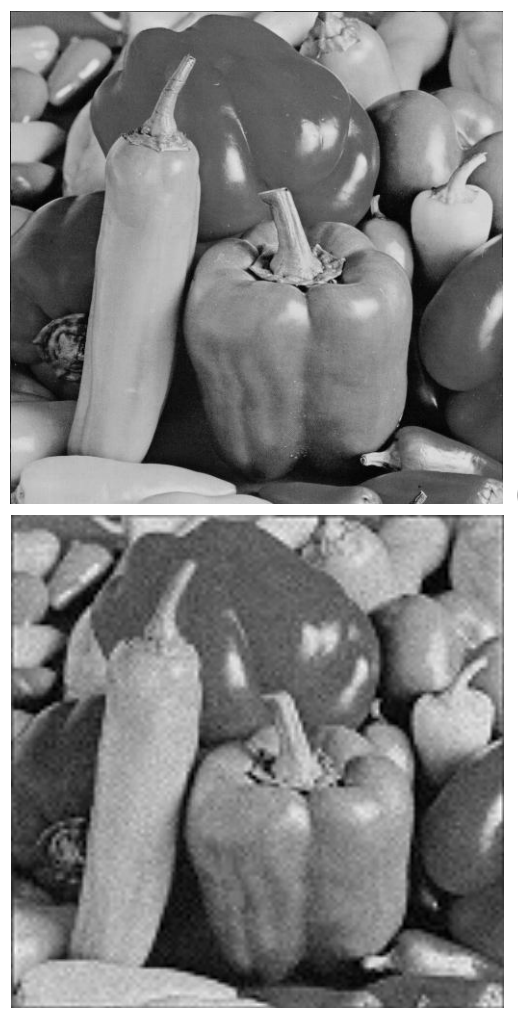

(a)

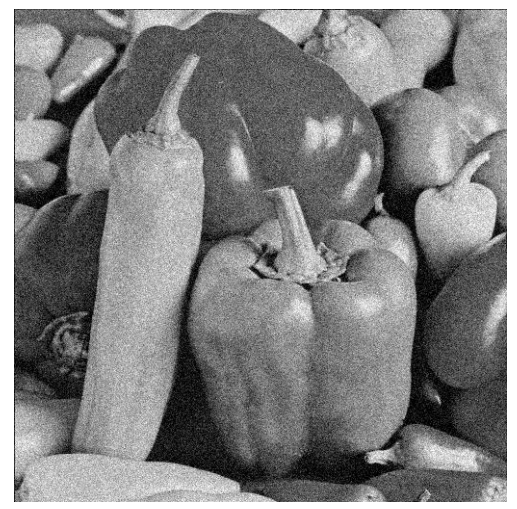

(b)

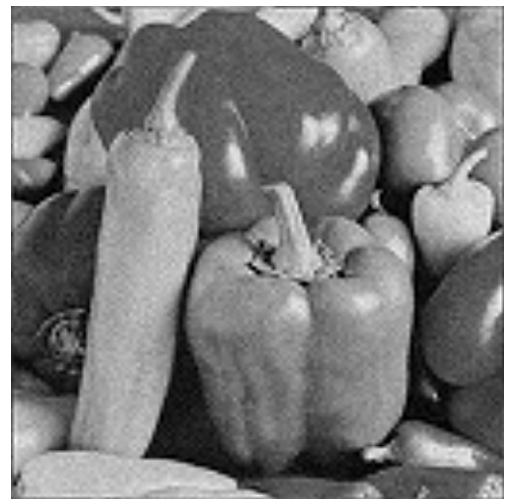

(c)

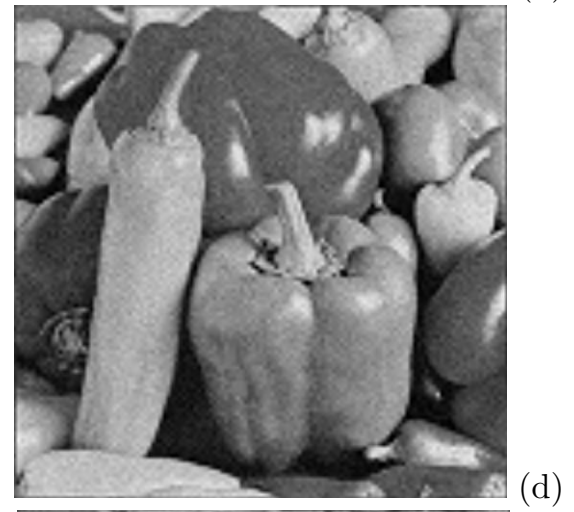

(e)

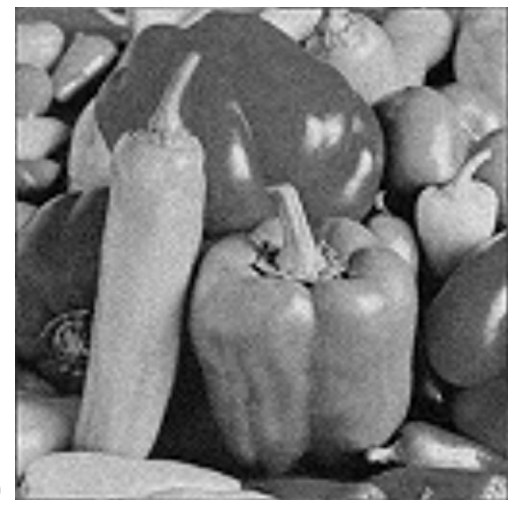

Figure 2: (a) Peppers image, (b) noise corrupted image (PSNR=21.73dB), (c) an image restored by the filters listed in Table $2(\mathrm{PSNR}=27.23 \mathrm{~dB}),(\mathrm{d})$ an image restored by the filters listed in Table $3(\mathrm{PSNR}=27.39 \mathrm{~dB}),(\mathrm{e})$ an image restored by the filters listed in Table $4(\mathrm{PSNR}=27.46 \mathrm{~dB})$, (f) an image restored by the filters listed in Table 5 $(\mathrm{PSNR}=27.64 \mathrm{~dB})$. 